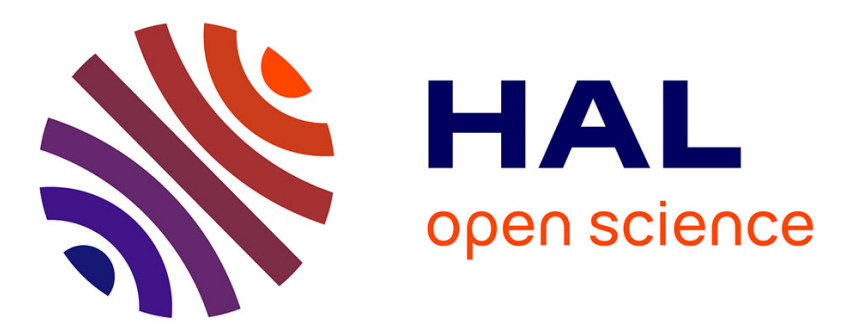

\title{
Improving reliability and efficiency of RTK ambiguity resolution using multiple rover receivers connected to the same antenna
}

Xiao Hu, Paul Thevenon, Christophe Macabiau

\section{- To cite this version:}

Xiao Hu, Paul Thevenon, Christophe Macabiau. Improving reliability and efficiency of RTK ambiguity resolution using multiple rover receivers connected to the same antenna. ENC 2020 European Navigation Conference, Nov 2020, Dresde ( Virtual event ), Germany. 10.23919/ENC48637.2020.9317367. hal-03147591

\section{HAL Id: hal-03147591 \\ https://hal-enac.archives-ouvertes.fr/hal-03147591}

Submitted on 20 Feb 2021

HAL is a multi-disciplinary open access archive for the deposit and dissemination of scientific research documents, whether they are published or not. The documents may come from teaching and research institutions in France or abroad, or from public or private research centers.
L'archive ouverte pluridisciplinaire HAL, est destinée au dépôt et à la diffusion de documents scientifiques de niveau recherche, publiés ou non, émanant des établissements d'enseignement et de recherche français ou étrangers, des laboratoires publics ou privés. 


\title{
Improving reliability and efficiency of RTK ambiguity resolution using multiple rover receivers connected to the same antenna
}

\author{
Xiao HU*, Paul THEVENON*, Christophe MABABIAU* \\ "Ecole Nationale de l'Aviation Civile, Université de Toulouse \\ Toulouse, FRANCE \\ Email: xiao.hu@recherche.enac.fr
}

\begin{abstract}
Precise position information is significantly required for specific application scenarios like autonomous driving of vehicles or precise mobile mapping. Until recently, to satisfy the sub-meter or even centimeter-level demanding for this type of application, the GNSS carrier phase measurements appear mandatory. In this paper, we firstly use multiple rover receivers to enhance the performance of the RTK positioning in different environments by connecting them to the same antenna thus multiplying the number of observations by the number of receivers. We demonstrate through different simulation scenarios that we are able to improve some internal steps of precise position computation and the simulations results show that our multi-receiver RTK system is more robust to noise and degraded satellite geometry, in terms of ambiguity fixing rate, and get a better position accuracy under same conditions when comparing to the single receiver system. An analysis is also conducted by varying the correlation coefficient of the noise between the measurements to the same satellite performed by the different receivers to verify the advantage of our proposed system when dealing with highly correlated noise.
\end{abstract}

\section{Introduction}

GNSS receivers have become a mass-market device by millions of users every day. Current positioning accuracy is usually sufficient to lead the way of a car driver into an unknown area or provide a stable timing signal to a mobile cell tower. However, stand-alone positioning technique is not precise enough for applications requiring sub-meter to centimeter-level accuracy such as precise mobile mapping and vehicles automatic control. To achieve this kind of level accuracy of positioning, the GNSS carrier phase measurements appear compulsory. Nevertheless, they are also less robust and include a so-called integer ambiguity resolution (IAR) that prevents them to be used directly for positioning. In some harsh environments, severe code multipath, frequent cycle slips, and losses of the lock might also challenge the process of finding the correct IAR thus reduce the accuracy.

To increase confidence and speed up the IAR process by limiting the search space, constraints can be established by using an array of two or more receivers with prior known and fixed geometry, including the length of the baseline vectors between the receivers of the array and the orientation of the vectors.

Recently, several studies have focused on the use of multiple receivers for attitude determination [1], [2] and calibration of magnetic field sensors [3], Daniel et al. [4] developed a method for the recursive estimation of the positioning and attitude problems using GNSS carrier phase observations from an array of receivers, but they calculated the position of each receiver separately thus they didn't take advantage of the known geometric constraints. Fan et al. [5] proposed a dual-antenna constraint RTK algorithm, which combines GNSS measurements of both antennas by making use of the geometric constraint between them, the reception diversity improves the availability and geometric distribution of GNSS satellites thus improves the positioning accuracy. However, their algorithm is severely restricted by the 
environmental conditions. Zheng et al. [6] presented a methodology for integrating carrier phase attitude determination and positioning systems by considering one of the receiver pairs in the attitude determination system also used as the rover for the relative positioning system. Nevertheless, their positioning systems remained independent which did not much ameliorate the success rate of IAR for the RTK positioning. Nandakumaran et al. [7] provided a numerical insight into the role taken by the multi-GNSS integration in delivering high-precision solutions, however, they focused on the PPP solution rather than the RTK processing which requires long convergence time to reach cm-level accuracy.

As far as we know, the use of an array of receivers is not used to improve the accuracy of the array position or for some internal steps of precise position computation. The concept of RTK positioning and attitude determination using an array of GNSS receivers with known geometry has already been introduced in [8]. In [9], we discussed the improvement of cycle-slip detection and repair for RTK processing by using an array of two receivers with known geometry.

In this contribution, we will focus on one specific scenario which is the improvement of RTK processing by using an array of two single-frequency receivers with zero baseline constraint which can be realized by connecting the receivers to the same antenna. The approach presented here aims at developing a method that takes full advantage of the measurement redundancy and diversity, along with the geometric constraints, between two closely placed rover antennas to improve the accuracy of the float ambiguity estimation and, consequently, the AR success rate.

An analysis is also conducted by varying the correlation coefficient of the noise between the measurements to the same satellite performed by the different receivers to verify the advantage of our proposed system when dealing with highly correlated noise between multiple rover receivers.

\section{Mathematical Models}

In this work, a Kalman Filter (KF) based position determination algorithm is developed. The scenario considers a moving vehicle, on which two low-cost, single-frequency receivers are connected to one single antenna, whereas the third one serves as a reference station, with its position static and known.

In the following, the state and the measurement vectors are described along with the statetransition and measurement models.

\subsection{State transition model}

The state transition or state-space model describes how the states or parameters of the system vary over time based on a specific linear model. In our KF modeling, the state parameter transition between subsequent epochs is given by:

$$
\mathbf{x}_{n}=\boldsymbol{\phi}_{n-1} \mathbf{x}_{n-1}+\mathbf{w}_{n}
$$

where:

$\mathbf{x}_{n} \quad$ refers to the state vector at epoch $n$

$\boldsymbol{\phi}_{n-1}$ refers to the transition matrix of epoch $n-1$

$\mathbf{w}_{n}$ refers to the so-called system noise vector at epoch $n$

Together with the process noise vector, one can define the process noise covariance matrix as:

$$
\mathbf{Q}_{n}=E\left[\mathbf{w}_{n} \mathbf{w}_{n}^{\mathrm{T}}\right]
$$


This matrix has then the variances of the state parameter's estimates based on the system model.

The estimated parameters are collected inside the state vector. The state vector collects 3 vehicle state parameters and $2 *\left(N_{\text {sat }}-1\right)$ satellite state parameters which are: the 3D position of GNSS receiver 1 relative to GNSS receiver 3, double-difference phase ambiguity of the GNSS receiver pair 1-3, and the double-difference phase ambiguity of the receiver pair 2-3. which is given by:

$$
\mathbf{x}=\left[\begin{array}{lll}
\mathbf{b}_{13}^{T} & \mathbf{N}_{13}^{T} & \mathbf{N}_{23}^{T}
\end{array}\right]^{T}
$$

where:

$\mathbf{N}_{n p} \quad$ refers to the vector of the double-difference GPS integer ambiguity between receiver $n$ and $p$

$\mathbf{b}_{13}^{T} \quad$ refers to the relative position vector between reference receiver 3 and rover receiver 1

\section{Transition model for position related state parameters}

In our KF modeling, for the position related state parameters, we suppose the speed components are a zero-mean Gaussian process, which can be defined as:

where:

$$
\mathbf{b}_{13, n}=\mathbf{b}_{13, n-1}+\mathbf{w}_{13, n}
$$

$\mathbf{w}_{13}$ is a centered Gaussian vector with a covariance matrix $\mathbf{Q}_{13}$

\section{Transition model for satellite-related state parameters}

In the case of the satellite-related parameters, they are assumed all as constant over subsequent epochs with a very small noise compared to the position related state parameters.

where:

$$
\begin{aligned}
& \mathbf{N}_{13, n}=\mathbf{N}_{13, n-1}+\mathbf{w}_{a m b, n} \\
& \mathbf{N}_{23, n}=\mathbf{N}_{23, n-1}+\mathbf{w}_{a m b, n}
\end{aligned}
$$

$\mathbf{w}_{a m b, n}$ is a centered Gaussian variable with a covariance matrix $\mathbf{Q}_{a m b}=\sigma_{a m b}^{2} \mathbf{I}_{N_{s a t}-1}$ $\mathbf{I}_{N}$ is the identity matrix of size $N$

The resulting state transition matrix $\boldsymbol{\phi}$ is then given by a unit matrix and different values of process noise variance are added to complete the model.

$$
\boldsymbol{\Phi}=\left[\begin{array}{ccc}
1 & \cdots & 0 \\
\vdots & \ddots & \vdots \\
0 & \cdots & 1
\end{array}\right]
$$

And the corresponding process noise matrix $\mathbf{Q}$ is given as follows:

\subsection{Measurement model}

$$
\mathbf{Q}=\left[\begin{array}{ccc}
\mathbf{Q}_{13} & \mathbf{0}_{3 \times N_{s a t}-1} & \mathbf{0}_{3 \times N_{s a t}-1} \\
\mathbf{0}_{N_{s a t}-1 \times 3} & \mathbf{Q}_{a m b} & \mathbf{0}_{N_{s a t}-1} \\
\mathbf{0}_{N_{s a t}-1 \times 3} & \mathbf{0}_{N_{s a t}-1} & \mathbf{Q}_{a m b}
\end{array}\right]
$$

The measurement model describes how the individual sensor measurements are related to system states. For every epoch $\mathrm{n}$, the measurement vector $\mathbf{z}_{\boldsymbol{n}}$, which contains all measured values, can be described as a function of the state vector $\mathbf{x}_{n}$ as:

$$
\mathbf{z}_{\boldsymbol{n}}=\mathbf{h}_{n}\left(\mathbf{x}_{\boldsymbol{n}}\right)+\mathbf{v}_{n}
$$

with $\mathbf{h}_{n}$ is the function that relates one or more states with each measured value and $\mathbf{v}_{n}$ is the measurement noise vector, which describes the expected Gaussian noise of every measured value.

As for the process noise covariance matrix, the definition of the measurement noise covariance matrix follows as: 


$$
\mathbf{R}_{n}=E\left[\mathbf{v}_{n} \mathbf{v}_{n}^{\mathrm{T}}\right]
$$

In our model, the measurement vector comprises the following measured values: Doubledifference (DD) code phase measurement vector of receiver 1, DD code phase measurement vector of receiver 2, DD carrier phase measurement vector of receiver 1, and DD carrier phase measurement vector of receiver 2 .

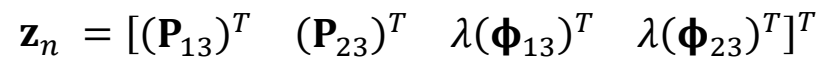

In this measurement model, the position of receiver 2 is assumed to share the same position as receiver 1.

The individual double-difference corrected pseudo-range and phase GPS measurement can be modeled as:

$$
\begin{gathered}
P_{13}^{k l}=\left(\mathbf{e}^{k l}\right)^{T} \mathbf{b}_{13}+n_{P, 13}^{k l} \\
P_{23}^{k l}=\left(\mathbf{e}^{k l}\right)^{T} \mathbf{b}_{13}+n_{P, 23}^{k l} \\
\lambda \phi_{13}^{k l}=\left(\mathbf{e}^{k l}\right)^{T} \mathbf{b}_{13}+\lambda N_{13}^{k l}+n_{\phi, 13}^{k l} \\
\lambda \phi_{23}^{k l}=\left(\mathbf{e}^{k l}\right)^{T} \mathbf{b}_{13}+\lambda N_{23}^{k l}+n_{\phi, 23}^{k l}
\end{gathered}
$$

where:

$P_{n p}^{k l} \quad$ is the double-difference code phase measurement of receiver pair $n p$ and satellite pair $k l$

$\lambda \phi_{n p}^{k l} \quad$ is the double-difference carrier phase measurement of receiver pair $n p$ and satellite pair $k l$

$\mathbf{e}^{k l} \quad$ is the difference between the Line of Sight vector of satellite $k$ and $l$

$N_{n p}^{k l} \quad$ is the double-difference integer ambiguity of receiver pair $n p$ and satellite pair $k l$

$n_{P, n p}^{k l}, n_{\phi, n p}^{k l}$ is the noise measurement of the double-difference code and phase measurement respectively

The LOS vectors are modeled by using the azimuth $\varphi_{k}$ and elevation $\theta_{k}$ of the satellites with respect to the corresponding receiver, thanks to the following formula who define the LOS vector between satellite $\mathrm{k}$ and the receiver in the ENU frame:

$$
\mathbf{e}^{k}=\left[\begin{array}{c}
\cos \theta_{k} \cos \varphi_{k} \\
\cos \theta_{k} \sin \varphi_{k} \\
\sin \theta_{k}
\end{array}\right]
$$

A fixed measurement noise variance between all satellites is defined to complete the measurement model, defining the measurement covariance matrix $\mathbf{R}$.

As the measurements are coming from signals received by the same antenna, it is safe to consider a certain level of correlation between these noise measurements. For example, the multipath error may be similar on the measurement performed by the 2 receivers from the same satellite.

The covariance coefficient between the 2 receivers is given by

$$
\operatorname{cov}_{12}^{k}=\rho_{12} \sigma_{1}^{k} \sigma_{2}^{k}
$$

Where

$\operatorname{cov}_{12}^{k}$ is the covariance of the noise affecting the measurement performed by receivers 1 and 2 on satellite $k$

$\rho_{12}$ is the correlation coefficient of the noise affecting the measurement performed by receivers 1 and 2

$\sigma_{n}^{k}$ is the standard deviation of the noise affecting the measurement performed by receiver $n$ on satellite $k$

We consider a simplifying assumption that the noise standard deviation does not depend on the satellite or the receivers $\left(\sigma_{n}^{k}=\sigma\right)$, and that the code measurement noise standard deviation and 
the phase measurement one are related by a simple factor $\sigma_{\text {carrier }}=k * \sigma_{\text {code }}$, with a value $k$ equals to $1 / 100$ to account for the much better accuracy of carrier phase measurements. The measurement covariance matrix $\mathbf{R}_{\mathrm{SD}}$ for the single difference measurements will have the following shape:

$$
\begin{gathered}
\mathbf{R}_{\text {code }, \mathrm{SD}}=2 * \sigma_{\text {code }}^{2}\left[\begin{array}{cc}
\mathbf{I}_{N_{\text {sat }}} & \rho_{12} \mathbf{I}_{N_{\text {sat }}} \\
\rho_{12} \mathbf{I}_{N_{\text {sat }}} & \mathbf{I}_{N_{\text {sat }}}
\end{array}\right] \\
\mathbf{R}_{\text {carrier }, \mathrm{SD}}=2 * k^{2} * \sigma_{\text {code }}^{2}\left[\begin{array}{cc}
\mathbf{I}_{N_{\text {sat }}} & \rho_{12} \mathbf{I}_{N_{\text {sat }}} \\
\rho_{12} \mathbf{I}_{N_{\text {sat }}} & \mathbf{I}_{N_{\text {sat }}}
\end{array}\right]
\end{gathered}
$$

The measurement covariance matrix $\mathbf{R}$ for the double-difference measurements can then be deduced using the following formula:

Where

$$
\mathbf{R}=\mathbf{D}\left[\begin{array}{cc}
\mathbf{R}_{\text {code,SD }} & \mathbf{0}_{N_{\text {sat }}} \\
\mathbf{0}_{N_{\text {sat }}} & \mathbf{R}_{\text {carrier }, \mathrm{SD}}
\end{array}\right] \mathbf{D}^{T}
$$

$$
\boldsymbol{D}=\left[\begin{array}{ccccc}
-1 & 0 & \cdots & 0 & 1 \\
0 & -1 & \ddots & \vdots & 1 \\
\vdots & \ddots & \ddots & 0 & \vdots \\
0 & \cdots & 0 & -1 & 1
\end{array}\right]
$$

is the single-differencing matrix used for computing the double-difference.

Two alternating steps which are the state prediction step and the state update step are then conducted to complete the KF algorithm.

\subsection{RTK processing}

In the previous process, we obtain a float estimation of the double-difference integer ambiguity. The accuracy of the position state estimate is further improved by fixing the DD ambiguities to integer number by using the well-known LAMBDA algorithm [10], [11]. This algorithm has shown its superiority in terms of both performance and processing time when compared to other algorithms in[12].

One selects the integer candidates based on the sum of squared errors to get a fixed solution. The candidate with the lowest error norm is chosen once the ratio of the Maximum A Posteriori error norm between the second-best candidate and the best candidate is bigger than a threshold. It is a pre-defined threshold or the critical value that the squared norm of ambiguity residuals of the best and second-best candidates should overpass to validate the integer estimation. In our paper, an empirical fixed value 3 is taken as in [13].

Once the IAR process is declared successful, a new position is computed using the DD carrier phase measurements corrected by the validated DD integer ambiguities. This final position is the fixed solution.

\section{Simulation of RTK performances using multiple rover receivers connected to the same antenna}

In this section, the proposed precise position determination algorithm is verified with simulated measurements. The reason why we use the simulated measurements is that we want to analyze the advantage of multiple receivers connected to the same antenna when varying the amplitude of the measurement noise, the level of noise correlation between the rover receivers' measurements and the satellite geometry. 
The vehicle is assumed to make a uniform circular motion around the fixed reference receiver 3 with a constant radius $(R=100 \mathrm{~m})$ in all the scenarios. The speed of the vehicle is $10 \mathrm{~m} / \mathrm{s}$, the duration of the simulated trajectory is $1000 \mathrm{~s}$ and the sampling rate is $1 \mathrm{~Hz}$.

In our simulator, the satellites are simulated with a fixed position. We consider 7 satellites with a fairly good geometric condition, i.e. well-spaced in azimuth and elevation. This would correspond to a typical case of a single constellation in an open sky environment. Finally, the DD carrier ambiguities are taken as fixed values separated by 10 cycles. This means that the DD ambiguities go from 10 to 120 .

Typical results of the state estimations including the estimation of the integer ambiguity by using LAMBDA are given in fig. 1.

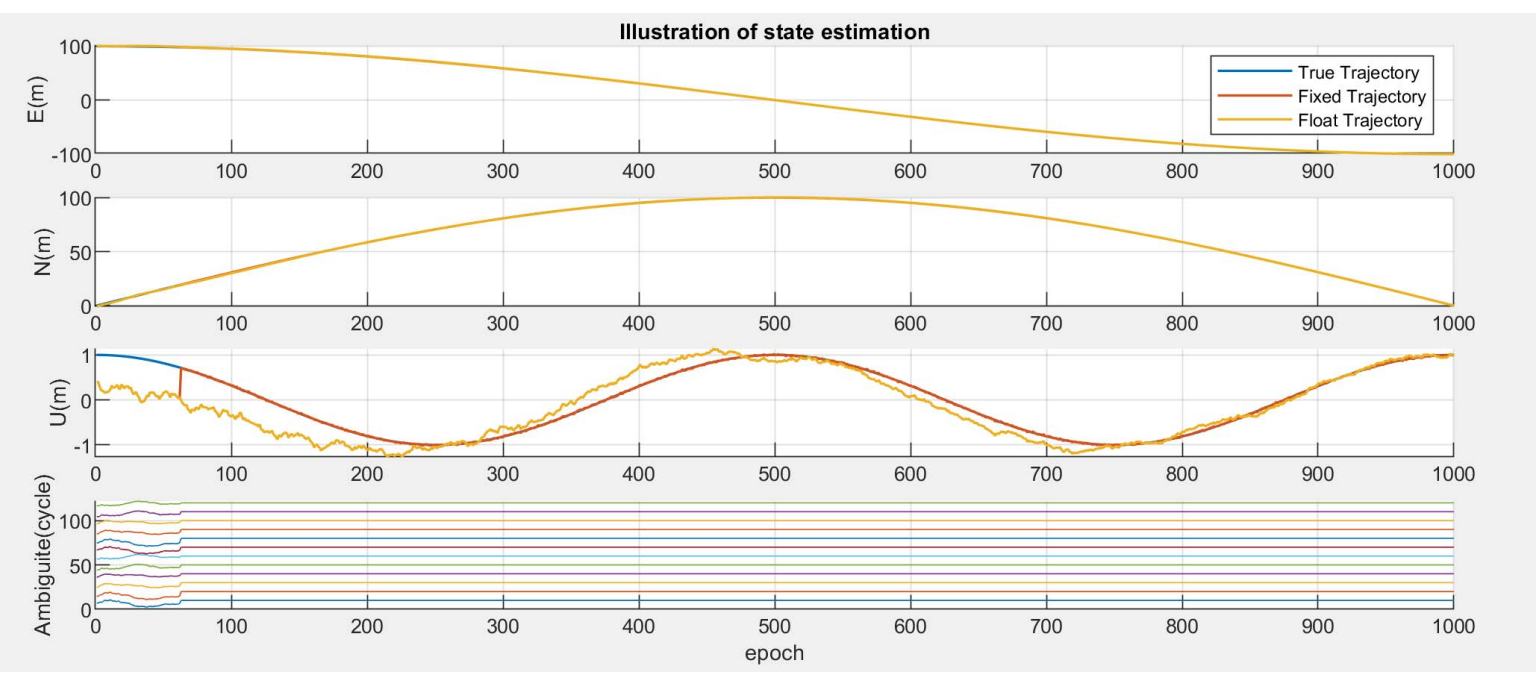

Figure 1. Illustration of state estimation including IAR

Fig. 2 gives the error comparison between the float and fixed solution. Here the fixed solution is defined by two parts, for every epoch, if the IAR process is declared successful, a new position is computed using the DD carrier phase measurements corrected by the validated DD integer ambiguities. This position then is used to update the fixed solution. If the IAR process is not declared successful, we keep the float solution.

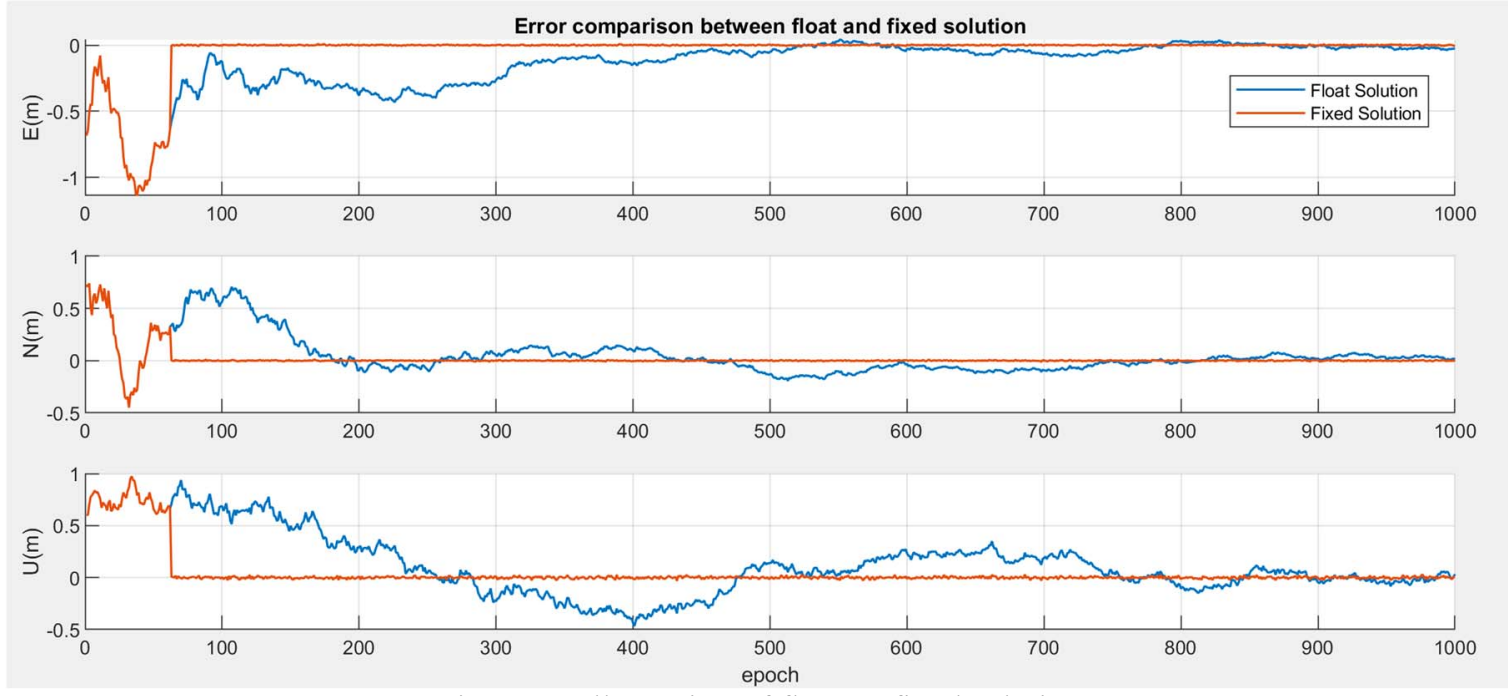

Figure 2. Illustration of float vs fixed solution

We can see from the fig.2 that the same convergence period is present in all states for the fixed solution. In the case of the float solution, the East, North orientations converge rapidly to the 
true value, however, due to the observability issue, the altitude takes more time to converge. What's more, once they converge, the error between the estimated result and the true value is extremely small especially in the case of the fixed solution. From the figure, one can notice that the system fixes the IAR in the 64th epoch and once the IAR is fixed, the positioning results become much more accurate compared to the float solution.

To validate the influence of the using of an array of receivers, several scenarios are conducted. Results are compared in terms of fixed rate and 3D position error statistics (mean, standard deviation, and $95 \%$ bound). The numbers have been computed by running 100 simulations with the different random realization of noise and initialization values and taking the average of the investigated indicators.

\subsection{Scenario 1: Geometry of satellite effect Analyses}

A favorable satellite geometry is essential to get an accurate positioning result. That is why in the first scenario, we want to analyze the influence of the system when the geometry of the satellite changes. In this scenario, both the correlation level $\left(\rho_{12}=0\right)$ and the amplitude of the noise measurement $\left(\sigma_{\text {code }}=1 \mathrm{~m}\right.$ ) are fixed and by degrading the geometry of the satellite (by removing one satellite at a time), we observe the robustness against degraded satellite geometry of multiple rover receivers RTK systems. The different scenarios are shown in fig. 3
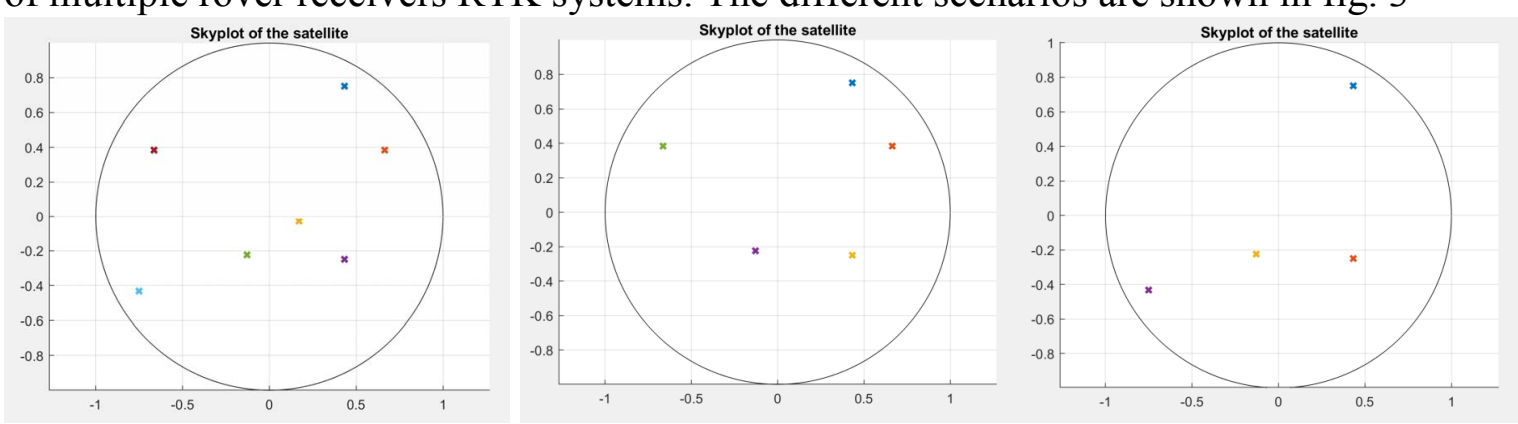

Figure 3. considered satellite geometry with $N_{\text {sat }}=7$ (left), $N_{\text {sat }}=5$ (middle) and $N_{\text {sat }}=4$ (right)

The analysis of Table 1 shows that the use of the receiver array improves the fixed rate for all considered satellite geometry, besides, the dual receiver array provides always better performance than the single receiver RTK solution under the same satellite geometry situation which confirms the advantage of our approach.

Table 1 - Performance comparison for different satellite geometry, $\rho_{12}=0, \sigma_{\text {code }}=1 \mathrm{~m}$

\begin{tabular}{|c|c|c|c|c|c|}
\hline $\begin{array}{c}\text { Variation of } \\
\text { scenario 1 }\end{array}$ & Performance indicator & Unit & Mean & $\begin{array}{c}\text { Standard } \\
\text { deviation }\end{array}$ & 95\% bound \\
\hline $\begin{array}{c}\text { Single receiver } \\
N_{\text {sat }}=7\end{array}$ & 3D position error & $\mathrm{m}$ & 0.1436 & 0.1975 & 0.0126 \\
\cline { 2 - 6 } & Fixed rate & $\%$ & 89.35 & 16.08 & 51.13 \\
\hline $\begin{array}{c}\text { Dual receiver } \\
N_{\text {sat }}=7\end{array}$ & 3D position error & $\mathrm{m}$ & 0.0323 & 0.1634 & 0.0014 \\
\cline { 2 - 6 } & Fixed rate & $\%$ & 98.59 & 7.98 & 96.76 \\
\hline Single receiver & 3D position error & $\mathrm{m}$ & 0.1839 & 0.2848 & 0.0148 \\
\cline { 2 - 6 }$N_{\text {sat }}=5$ & Fixed rate & $\%$ & 72.43 & 22.86 & 21.21 \\
\hline Dual receiver & 3D position error & $\mathrm{m}$ & 0.1431 & 0.2228 & 0.0026 \\
\cline { 2 - 6 }$N_{\text {sat }}=5$ & Fixed rate & $\%$ & 85.21 & 20.24 & 36.18 \\
\hline Single receiver & 3D position error & $\mathrm{m}$ & 0.6264 & 0.3324 & 0.4812 \\
\cline { 2 - 6 }$N_{\text {sat }}=4$ & Fixed rate & $\%$ & 0.82 & 0.49 & 0.11 \\
\hline
\end{tabular}




\begin{tabular}{|c|c|c|c|c|c|}
\hline Dual receiver & 3D position error & $\mathrm{m}$ & 0.3298 & 0.2881 & 0.1736 \\
\cline { 2 - 6 }$N_{\text {sat }}=4$ & Fixed rate & $\%$ & 34.71 & 27.80 & 7.85 \\
\hline
\end{tabular}

\subsection{Scenario 2: Noise measurement effect Analyses}

In the second scenario, the correlation level of the noise measurement $\left(\rho_{12}=0\right)$ and the satellite geometry $\left(N_{s a t}=7\right)$ are fixed, by varying the amplitude of the noise measurement, we observe the robustness against the noise of the multi-receiver RTK system.

We recall that the phase measurement noise standard deviation is scaled by a factor $1 / 100$, to account for the improved accuracy of the carrier phase measurement, compared to the code measurement. The analysis of Table 2 shows that the use of the receiver array improves the fixed rate for all considered $\sigma_{\text {code }}$ values, thus demonstrating the interest of such an approach.

Table 2 - Performance comparison for different measurement noise, $\rho_{12}=0, N_{\text {sat }}=7$

\begin{tabular}{|c|c|c|c|c|c|}
\hline $\begin{array}{c}\text { Variation of } \\
\text { scenario } 2\end{array}$ & Performance indicator & Unit & Mean & $\begin{array}{c}\text { Standard } \\
\text { deviation }\end{array}$ & 95\% bound \\
\hline Single receiver & 3D position error & $\mathrm{m}$ & 0.1436 & 0.1975 & 0.0126 \\
\cline { 2 - 6 }$\sigma_{\text {code }}=1 \mathrm{~m}$ & Fixed rate & $\%$ & 89.35 & 16.08 & 51.13 \\
\hline Dual receiver & 3D position error & $\mathrm{m}$ & 0.0323 & 0.1634 & 0.0014 \\
\cline { 2 - 6 }$\sigma_{\text {code }}=1 \mathrm{~m}$ & Fixed rate & $\%$ & 98.59 & 7.98 & 96.76 \\
\hline Single receiver & 3D position error & $\mathrm{m}$ & 0.1815 & 0.2672 & 0.0076 \\
\cline { 2 - 6 }$\sigma_{\text {code }}=2 \mathrm{~m}$ & Fixed rate & $\%$ & 66.25 & 18.04 & 28.82 \\
\hline Dual receiver & 3D position error & $\mathrm{m}$ & 0.0678 & 0.1561 & 0.0045 \\
\cline { 2 - 6 }$\sigma_{\text {code }}=2 \mathrm{~m}$ & Fixed rate & $\%$ & 83.61 & 18.48 & 33.92 \\
\hline Single receiver & 3D position error & $\mathrm{m}$ & 0.4355 & 0.3584 & 0.3410 \\
\cline { 2 - 6 }$\sigma_{\text {code }}=10 \mathrm{~m}$ & Fixed rate & $\%$ & 15.03 & 3.09 & 9.26 \\
\hline Dual receiver & 3D position error & $\mathrm{m}$ & 0.2162 & 0.2287 & 0.0108 \\
\cline { 2 - 6 }$\sigma_{\text {code }}=10 \mathrm{~m}$ & Fixed rate & $\%$ & 49.08 & 14.42 & 20.98 \\
\hline
\end{tabular}

\subsection{Scenario 3: Noise measurement Correlation effect Analyses}

In probability theory and statistics, correlation (or correlation coefficient) is used as a measure of the linear correlation between two variables $X$ and $Y$. The most widely used is the Pearson product-moment correlation coefficient (PPMCC). It is defined as the covariance of two variables divided by the standard deviation of the two variables as shown in (17)

$$
\rho_{X, Y}=\frac{\operatorname{cov}(X, Y)}{\sigma_{X} \sigma_{Y}}=\frac{E\left[\left(X-\mu_{X}\right)\left(Y-\mu_{Y}\right)\right]}{\sigma_{X} \sigma_{Y}}
$$

The significance of the magnitude of the correlation coefficient is currently inconsistent in the statistical world, but it is usually considered as follows:

Table 3 - Significance of the magnitude of the correlation coefficient

\begin{tabular}{|c|l|}
\hline $0.00- \pm 0.30$ slightly correlated & $\pm 0.30- \pm 0.50$ medium correlation \\
\hline $\pm 0.50- \pm 0.80$ significantly correlated & $\pm 0.80- \pm 1.00$ highly correlated \\
\hline
\end{tabular}

As we discussed in the previous section, in our model, the measurements are coming from signals received by the same antenna, thus it is paramount to consider a certain level of 
correlation of these noise measurements to make it closer to the real situation. As an example, the multipath error may be similar on the measurements performed by the 2 receivers from the same satellite.

Consequently, in this scenario, we want to verify what level of correlation is acceptable to keep an improvement compared to a single receiver RTK system. The correlation level of the noise measurement is not fixed, by varying the noise correlation coefficient while keeping the same noise measurement amplitude $\left(\sigma_{\text {code }}=1 \mathrm{~m}\right)$ and a favorable satellite geometry $\left(N_{\text {sat }}=7\right)$. The different scenarios of the magnitude of the correlation coefficient are shown in Fig. 4
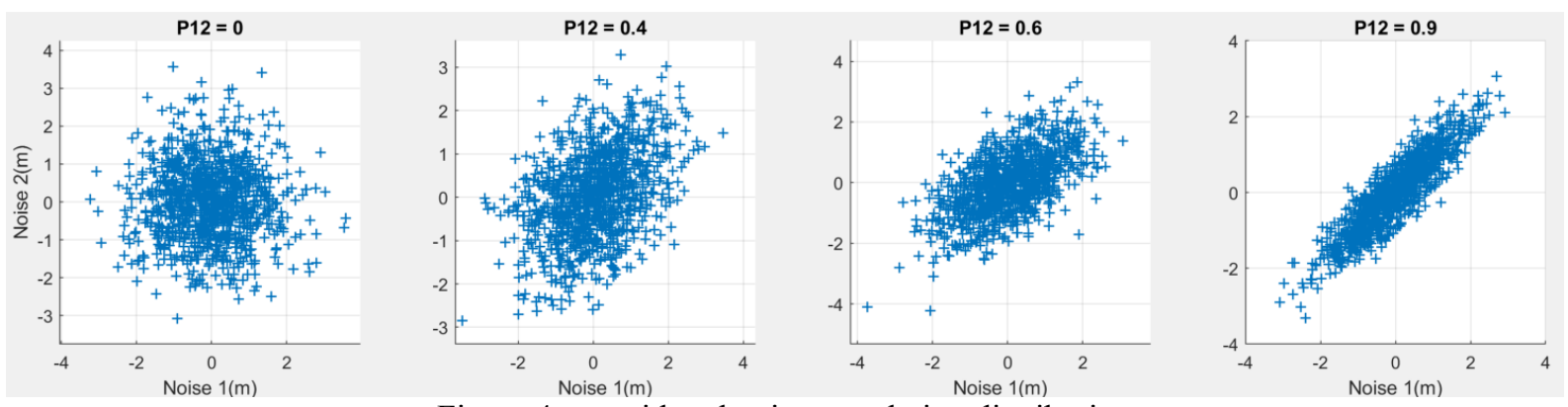

Figure 4. considered noise correlation distribution

The result is shown in Table 4, from the result one can conclude that the dual receiver RTK system provides always better performance than the single receiver RTK solution and the position accuracy sightly improves as the magnitude of the correlation coefficient decreases. In addition, the fixing rate and positioning accuracy are not significantly reduced even when dealing with highly correlated noise, which proves that our system is exceedingly robust and stable when handling high correlation noise situations. This may be due to the fact that the LAMBDA algorithm takes into account the correlation of the measurements in the IAR process, which is assumed perfectly known in our simulation.

Table 4 - Performance comparison for different measurement noise, $\sigma_{\text {code }}=1 \mathrm{~m}, N_{\text {sat }}=7$

\begin{tabular}{|c|c|c|c|c|c|}
\hline $\begin{array}{c}\text { Variation of } \\
\text { scenario 3 }\end{array}$ & Performance indicator & Unit & Mean & $\begin{array}{c}\text { Standard } \\
\text { deviation }\end{array}$ & 95\% bound \\
\hline \multirow{2}{*}{ Single receiver } & 3D position error & $\mathrm{m}$ & 0.1436 & 0.1975 & 0.0126 \\
\cline { 2 - 6 } & Fixed rate & $\%$ & 89.35 & 16.08 & 51.13 \\
\hline \multirow{2}{*}{$\begin{array}{c}\text { Dual receiver } \\
\rho_{12}=0\end{array}$} & 3D position error & $\mathrm{m}$ & 0.0323 & 0.1634 & 0.0014 \\
\cline { 2 - 6 } Dual receiver & Fixed rate & $\%$ & 98.59 & 7.98 & 96.76 \\
\cline { 2 - 6 }$\rho_{12}=0.4$ & 3D position error & $\mathrm{m}$ & 0.0353 & 0.1822 & 0.0014 \\
\hline Dual receiver & Fixed rate & $\%$ & 97.30 & 8.08 & 95.84 \\
\cline { 2 - 6 }$\rho_{12}=0.6$ & Fixed rate & $\%$ & 96.40 & 8.25 & 95.46 \\
\hline $\begin{array}{c}\text { Dual receiver } \\
\rho_{12}=0.9\end{array}$ & 3D position error & $\mathrm{m}$ & 0.0388 & 0.1930 & 0.0015 \\
\cline { 2 - 6 } & Fixed rate & $\%$ & 95.40 & 8.43 & 95.23 \\
\hline $\begin{array}{c}\hat{\rho}_{12}=0.4 \\
\rho_{12}=0.9\end{array}$ & 3D position error & $\mathrm{m}$ & 0.0392 & 0.1948 & 0.0015 \\
\cline { 2 - 6 } & Fixed rate & $\%$ & 95.30 & 8.37 & 95.14 \\
\hline
\end{tabular}

For verifying the sensitivity of the LAMBDA algorithm to a correct correlation coefficient, the final simulation has been conducted, where the correlation coefficient considered in the 
algorithm is different from the one used to generate the measurement ( $\hat{\rho}_{12}=0.4$ for all the simulations), a similar result is obtained as shown in the last line of table 4.

\section{Conclusion}

In this contribution, the method using multiple receivers connected to the same antenna (zerobaseline configuration) for the RTK positioning in different environments is studied. Taking advantage of the doubled quantity of the observations, we are able to improve some internal steps of precise position computation. We demonstrate through simulations results that our multi-receiver RTK system is more robust to noise and degraded satellite geometry, in terms of ambiguity fixing rate, and get a better position accuracy under the same conditions when comparing with the single receiver system. Furthermore, the mitigation of high correlation noise between receivers is permitted by using our system.

\section{References}

[1] M. Iafrancesco, "GPS/INS Tightly coupled position and attitude determination with low-cost sensors Master Thesis," p. 69.

[2] P. Henkel and C. Gunther, "Attitude determination with low-cost GPS/ INS," p. 9.

[3] P. Henkel, P. Berthold, and J. J. Kiam, "Calibration of magnetic field sensors with two massmarket GNSS receivers," in 2014 11th Workshop on Positioning, Navigation and Communication (WPNC), Dresden, Germany, Mar. 2014, pp. 1-5, doi: 10.1109/WPNC.2014.6843306.

[4] D. Medina, A. Heselbarth, R. Buscher, R. Ziebold, and J. Garcia, "On the Kalman filtering formulation for RTK joint positioning and attitude quaternion determination," in 2018 IEEE/ION Position, Location and Navigation Symposium (PLANS), Monterey, CA, Apr. 2018, pp. 597-604, doi: 10.1109/PLANS.2018.8373432.

[5] Fan, Li, Cui, and Lu, "Precise and Robust RTK-GNSS Positioning in Urban Environments with Dual-Antenna Configuration," Sensors, vol. 19, no. 16, p. 3586, Aug. 2019, doi: $10.3390 / \mathrm{s} 19163586$.

[6] G. Zheng and D. Gebre-Egziabher, "Enhancing Ambiguity Resolution Performance Using Attitude Determination Constraints," presented at the ION GNSS, Savannah, Georgia, Sep. 2009

[7] N. Nadarajah, A. Khodabandeh, K. Wang, M. Choudhury, and P. Teunissen, "Multi-GNSS PPPRTK: From Large- to Small-Scale Networks," Sensors, vol. 18, no. 4, p. 1078, Apr. 2018, doi: $10.3390 / \mathrm{s} 18041078$.

[8] X. Hu, P. Thevenon, and C. Macabiau, "Improvement of RTK performances using an array of receivers with known geometry," presented at the ITM 2020, International Technical Meeting, Jan. 2020, doi: 10.33012/2020.17154.

[9] X. Hu, P. Thevenon, and C. Macabiau, "Cycle-slip Detection and Repair Using an Array of Receivers with Known Geometry for RTK Positioning," PLANS 2020 IEEE/ION Position, Location and Navigation Symposium, Apr 2020, Portland, United States. pp.1123-1134 / ISBN: 978-1-7281- 9446-2, ff10.1109/PLANS46316.2020.9109871f

[10] P. J. G. Teunissen, "The least-squares ambiguity decorrelation adjustment: a method for fast GPS integer ambiguity estimation," J. Geod., vol. 70, no. 1-2, pp. 65-82, Nov. 1995, doi: 10.1007/BF00863419.

[11] P. Buist, "The Baseline Constrained LAMBDA Method for Single Epoch, Single Frequency Attitude Determination Applications," presented at the ION GNSS, Fort Worth, Texas, Sep. 2007,

[12] L. Baroni and H. K. Kuga, "Analysis of Attitude Determination Methods Using GPS Carrier Phase Measurements," Math. Probl. Eng., vol. 2012, pp. 1-10, 2012, doi: 10.1155/2012/596396.

[13] P. Teunissen and S. Verhagen, "On the Foundation of the Popular Ratio Test for GNSS Ambiguity Resolution," presented at the ION GNSS, Long Beach, California, Sep. 2004, Accessed: May 26, 2016. 